\title{
Symmetry Processing in the Macaque Visual Cortex
}

\author{
Pauline Audurier (1,2, Yseult Héjja-Brichard(101,2, Vanessa De Castro(1) ${ }^{1,2}$, \\ Peter J. Kohler ${ }^{3,4}$, Anthony M. Norcia ${ }^{5,6}$, Jean-Baptiste Durand ${ }^{1,2}$ and \\ Benoit R. Cottereau ${ }^{1,2}$
}

${ }^{1}$ Centre de Recherche Cerveau et Cognition, Université de Toulouse, 31052 Toulouse, France, ${ }^{2}$ Centre National de la Recherche Scientifique, 31055 Toulouse, France, ${ }^{3}$ Department of Psychology, York University, Toronto, ON M3J 1P3, Canada, ${ }^{4}$ Centre for Vision Research, York University, Toronto, ON, M3J 1P3, Canada, ${ }^{5}$ Department of Psychology, Stanford University, Stanford, CA 94305, USA and ${ }^{6} \mathrm{Wu}$ Tsai Neurosciences Institute, Stanford University, Stanford, CA 94305, USA

Address correspondence to Pauline Audurier. Email: pauline.audurier@cnrs.fr and Benoit R. Cottereau. Email: benoit.cottereau@cnrs.fr

\begin{abstract}
Symmetry is a highly salient feature of the natural world that is perceived by many species. In humans, the cerebral areas processing symmetry are now well identified from neuroimaging measurements. Macaque could constitute a good animal model to explore the underlying neural mechanisms, but a previous comparative study concluded that functional magnetic resonance imaging responses to mirror symmetry in this species were weaker than those observed in humans. Here, we re-examined symmetry processing in macaques from a broader perspective, using both rotation and reflection symmetry embedded in regular textures. Highly consistent responses to symmetry were found in a large network of areas (notably in areas V3 and V4), in line with what was reported in humans under identical experimental conditions. Our results suggest that the cortical networks that process symmetry in humans and macaques are potentially more similar than previously reported and point toward macaque as a relevant model for understanding symmetry processing.
\end{abstract}

Key words: fMRI, macaque, nonhuman primate, symmetry, texture

\section{Introduction}

As written by the physicist and essayist Alan Lightman: "The deep question is: Why does nature embody so much symmetry? We do not know the full answer to this question" (Lightman 2013). The 4 fundamental forms of symmetry in the plane (reflection, rotation, translation, and glide reflection) and their combinations are prevalent in natural scenes, which may have put evolutionary pressure on the visual systems of multiple species to facilitate their sensory processing of symmetry and its perception. Many animals, such as birds (Delius and Nowak 1982), fishes (Merry and Morris 2001), or even insects (Giurfa et al. 1996), are indeed able to detect symmetry, notably as a marker of phenotypic and/or genotypic quality in potential partners (Møller 1992). In humans, perception of symmetry in the 2D planes has been well documented by psychologists (see Wagemans 1997; Bertamini et al. 2018 for a review), and it has been suggested that symmetry contributes to viewpoint invariant representation of objects (Li et al. 2013) and to the encoding of regularity and structure in visual scenes (Liu et al. 2010; Kohler et al. 2016; Kohler and Clarke 2021). It could also underlie some aspects of our aesthetic judgments (Jacobsen and Höfel 2003) as demonstrated by its omnipresence in art, craft, and architecture.

The neural mechanisms underlying symmetry processing have been most extensively studied in humans using functional magnetic resonance imaging (fMRI) data and event-related potentials, with the focus being predominantly on reflection symmetry in the fronto-parallel plane (Sasaki et al. 2005; Van

○ The Author(s) 2021. Published by Oxford University Press. All rights reserved. For permissions, please e-mail: journals.permissions@oup.com This is an Open Access article distributed under the terms of the Creative Commons Attribution Non-Commercial License (http://creativecommons.org/ licenses/by-nc/4.0/), which permits non-commercial re-use, distribution, and reproduction in any medium, provided the original work is properly cited. For commercial re-use, please contact journals.permissions@oup.com 
Meel et al. 2019), although some studies also explored rotation and translation symmetry (Makin et al. 2013) or reflection symmetry in slanted planes (Makin et al. 2014; Keefe et al. 2018) Recent fMRI and electroencephalography (EEG) studies from our group (Kohler et al. 2016; Kohler and Clarke 2021) have used a class of regular textures known as wallpaper groups in which the 4 fundamental forms of symmetry (reflection, rotation, translation, and glide reflection) are combined and repeated to tile the plane (Fedorov 1891). This work demonstrates that human visual cortex carries highly detailed representations of the different symmetry types and their combinations. Because symmetries are a form of image redundancy, responses to wallpaper groups may reflect an extension of the principle of efficient coding to symmetries in images (Kohler et al. 2016; Kohler and Clarke 2021). Functional MRI measurements have made it possible to determine where in the brain these operations take place. Symmetry selective responses were observed in extrastriate visual areas like V3 and V4 as well as higher-level regions in both the dorsal (e.g., area V3A) and ventral (e.g., areas VO1, VO2, or LO) pathways (Sasaki et al. 2005; Kohler et al. 2016; Keefe et al. 2018; Van Meel et al. 2019). While these studies have provided important information regarding how and where different forms of symmetry are represented in the cortical hierarchy, they cannot address questions of the underlying cellular mechanisms involved in symmetry processing. In this context, macaques could constitute a promising animal model because they perceive symmetry (Waitt and Little 2006), and it is established that the functional organization of their visual system is substantially similar to that of humans (Felleman and Van Essen 1991; Orban et al. 2004). To assess the utility of a macaque model for symmetry processing in humans, it is important to first determine whether the cortical areas with significant responses to symmetry are analogous in these 2 primate species.

So far, only one study has explored symmetry processing in humans and monkeys using a comparative approach. In this study, individuals of both species were exposed to random dot patterns with (or without) reflection symmetry (Sasaki et al. 2005). Other forms of symmetry were not considered. Extensive measurements using standard experimental conditions at 3 Teslas failed to reveal significant responses to reflection symmetry in macaque. Only by using contrast agents or high-field fMRI at 7 Teslas could the authors detect symmetry-related activations in this species. These activations were only observed within areas V4d and V3A, suggesting a much more restricted cortical network than the one observed in humans using the same protocol. The fact that symmetry was found to evoke weak cortical responses in macaque possibly discouraged further explorations (notably in electrophysiology) in that model. To our knowledge, there has been no study on symmetry processing in the nonhuman primate brain since this single publication, more than 15 years ago.

Here, we re-examined the fMRI responses to symmetry in macaque from a broader perspective. We used different types of wallpaper groups (Liu et al. 2010) to determine whether the amount of symmetry in a given texture modulates cortical activations, as observed in humans (Sasaki et al. 2005; Kohler et al. 2016). To facilitate the comparison between the 2 primate species, we reproduce the experimental protocol of the study by Kohler et al. (2016). Our results clearly establish that macaque brains process symmetry using a much broader cortical network than previously documented and that the areas involved are closely related to those observed in humans. Thus, the present study calls for a re-examination of the relevance of the macaque model for symmetry processing in humans, and it opens the door to a characterization of the underlying neural mechanisms at the single-cell level, notably in area V3.

\section{Materials and Methods}

\section{Subjects}

Two female rhesus macaques M01 and M02 (age: 17-18 years; weight: $5.30-5.80 \mathrm{~kg}$ ) were involved in this study. This project was approved by the French Ministry of Research (MP/03/34/10/09) and a local ethics committee (CNREEA code: C2EA - 14). The housing and all the experimental protocols such as surgery, behavioral training, and fMRI recordings (see details Cottereau et al. 2017) were conducted with respect of the European Union legislation (2010/63/UE) and of the French Ministry of Agriculture guidelines (décret 2013-118). As required and recommended for primate welfare, the 2 animals were housed together in a social group of 4 individuals into a spacious and enriched enclosure and could thereby develop species-specific behavior such as foraging and congeners delousing.

\section{Visual Stimuli with Rotation or Reflection Symmetry}

Our stimuli were defined from previous mathematical works on symmetry based on wallpaper patterns (Fedorov 1891; Liu et al. 2010). Wallpaper patterns are repetitive 2D patterns that tile the plane. There are 17 unique wallpaper patterns, which cover all planar symmetry groups. Each group is built from a "unit lattice" that is used for tiling the plane without gaps. The "fundamental region" is the smallest repeating region in the wallpaper patterns. Within the unit lattice, multiple rigid transformations are applied to the fundamental region, which give rise to symmetries within the wallpaper group. Each wallpaper group contains a distinct combination of 4 fundamental symmetries: translations, rotations, reflections, and glide reflections (see Kohler et al. 2016 for more details). In our experiments, wallpaper patterns were formed by square images of 7 by 7 unit lattices of $1.7^{\circ}$ by $1.7^{\circ}$. These square images were subsequently cropped by a circular aperture (11.9 of diameter). In order to characterize the cortical responses to different types of symmetry in macaque, we used here stimuli with either rotation (experiment 1) or reflection (experiment 2) symmetries. The rotation symmetry stimuli were identical to those used in a previous human fMRI study (Kohler et al. 2016) and belonged to 4 different wallpaper groups: P2, P3, P4, and P6. All 4 groups contained translation and rotation symmetry but differed in the maximum number of rotations that left the stimuli unchanged. Indeed, rotation symmetry around a point can be defined in terms of its order $n$, where a rotation by an angle of $360 / n$ does not modify the stimuli. Stimuli from P2, P3, P4, and P6 groups are therefore, respectively, invariant to rotation of 180 , 120,90 , and $60^{\circ}$. Stimuli were generated from a noise texture in which a "fundamental region" was first defined and then repeated and rotated around several points, according to the group's order of rotation that they belong to (see Fig. 1A). The reflection symmetry stimuli were generated using the same procedure but belong to 2 distinct wallpaper groups, PM and PMM. Both contain reflection and translation symmetry, but while PM has reflection symmetry axes only in 1 direction, PMM contains axes in 2 orthogonal directions. We generated versions of PM that had either horizontal or vertical axes of reflection and labeled them PM_v (vertical) and PM_h (horizontal). PMM had reflection axes in both the vertical and horizontal directions 
(see Fig. 1B). For each stimulus exemplar within each wallpaper group (P2, P3, P4, P6, PM_h, PM_v, and PMM), we defined a control by applying a 2D Fourier transform, scrambling the phases of the Fourier coefficients at each frequency, and computing the inverse Fourier transform (see Fig. 1A). This operation leaves the amplitude spectrum unchanged and therefore preserves the stimulus local properties (luminance, orientation, spatial frequency, etc). It nonetheless disrupts the symmetry content and controls thereby always degenerate to the simplest wallpaper group, P1, which only contains translation symmetry but no rotation or reflection symmetry.

\section{MRI Recordings}

Recordings were performed using a 3 Tesla clinical MR scanner (Philips Achieva) and a dedicated custom 8-channel phased array coil (RapidBiomed) specially designed to fit with the macaque head shape while preserving their field of view.

\section{Recordings for Individual Templates}

Individual structural and functional templates were estimated for our 2 animals from recording sessions acquired under a slight anesthesia (Zoletil 100:10 mg/kg and Domitor: $0.04 \mathrm{mg} / \mathrm{kg}$ ) controlled with an MR compatible oximeter. These recordings consisted in $4 \mathrm{~T} 1$-weighted anatomical volumes at high resolution (magnetization prepared rapid gradient echo [MPRAGE]; repetition time, $\mathrm{TR}=10.3 \mathrm{~ms}$; echo time, $\mathrm{TE}=4.6 \mathrm{~ms}$, flip angle $=8^{\circ}$; FOV: $155 \times 155 \mathrm{~mm}$; matrix size: $312 \times 192 \mathrm{~mm}$; voxel size $=0.5 \times 0.5 \times 0.5 \mathrm{~mm} ; 192$ sagittal slices acquired in an interleaved order) and 300 functional volumes (gradientecho EPI; TR $=2000 \mathrm{~ms}, \mathrm{TE}=30 \mathrm{~ms}$, flip angle $=75^{\circ}$, SENSE factor =1.6; FOV: $100 \times 100 \mathrm{~mm}$; matrix size: $68 \times 64 \mathrm{~mm}$; voxel size $=1.25 \times 1.25 \times 1.5 \mathrm{~mm}, 32$ axial slices acquired in an interleaved order with a thickness of $1.5 \mathrm{~mm}$ and no gap). Anatomical and functional individual templates were derived from those volumes using a procedure that is described in detail in Cottereau et al. (2017).

\section{Functional Recordings}

fMRI recordings were conducted on awake behaving animals on a daily basis and lasted about an hour ( $\sim 10$ runs). The animals were head-fixed, seated in a sphinx position within their primate chair, facing an LCD screen (field of view: $11.9^{\circ}$ $\times 11.9^{\circ}$, resolution: 900 by 900 pixels) at a viewing distance of $1.25 \mathrm{~m}$. They were involved in a passive fixation task while the position of one eye was monitored with an infrared video-based eye-tracker at $500 \mathrm{~Hz}$ (Cambridge Research) placed on top of the primate chair. They were water-rewarded during correct fixation (i.e., when their gaze was within a circle of $1^{\circ}$ radius around the central fixation point).

For each symmetry condition (P2, P3, P4, and P6 in experiment 1 and PM_h, PM_v, and PMM in experiment 2), the main stimuli and their corresponding controls were presented using a block design. Each run consisted of $234 \mathrm{~s}$ (117 TRs) divided into 3 identical cycles of $72 \mathrm{~s}$ (36 TRs) plus an additional baseline of $18 \mathrm{~s}$ (9 TRs) during which only the fixation point was present. Each cycle started with a baseline of $18 \mathrm{~s}$ (9 TRs) during which only a gray screen was presented (its luminance was equal to the average luminance in the symmetry stimuli and in their controls). In half of the runs, it was followed by a block of $18 \mathrm{~s}$ with symmetric stimuli, then by another $18 \mathrm{~s}$ of baseline and finally by a block of $18 \mathrm{~s}$ with control stimuli. During a 18-s block, a new stimulus appeared every $500 \mathrm{~ms}$, and therefore, there were 36 different stimuli in total. In the other half of the runs, the sequence was reversed and the first baseline of the cycles was followed by a block of control stimuli. Both types of runs were intermixed. The 36 control stimuli in the control blocks corresponded to the 36 stimuli in the symmetric block of the same cycle. The whole experiment (i.e., visual display, eye monitoring, and water reward) was controlled using the EventIDE software (Okazolab). Data from the 2 experiments were collected separately.

\section{Data Processing}

\section{Individual Templates of Reference}

Data collected during the anesthetized sessions (see above) were used to estimate individual functional and anatomical templates. The anatomical template was obtained by realigning and averaging the $4 \mathrm{~T} 1$-weighted (MPRAGE) volumes. It was then aligned to the Montreal Neurological Institute space of the 112RM-SL template (see McLaren et al. 2009, 2010). Cortical surface reconstructions were performed using the CARET software (Van Essen et al. 2001). The functional template was obtained by realigning and averaging the 300 functional (GE-EPI) volumes. It was aligned with the anatomical template. Spatial normalization parameters (affine and non-rigid) between the functional and anatomical templates were determined from the gray matter maps of both templates. For group analyses, the same operation was performed to register each individual anatomical template to the F99 template available in the CARET software (Van Essen 2002).

\section{Preprocessing of the Functional Data}

To minimize the influence of eye movements on our results, only runs with high fixation rate $(>85 \%$ ) were considered for further analyses. For our 2 monkeys (M01 and M02), we respectively collected 16 and 25 of such runs for each rotation symmetry groups (i.e., P2, P3, P4 and P6) in the first experiment. We also collected 18 (M01) and 16 (M02) of such runs for each reflection symmetry group (PM_h, PM_v, and PMM) in the second experiment. For each experiment, the different symmetry conditions were interleaved between runs. The 4 first volumes of each run were removed to account for signal stabilization. The remaining 113 volumes were then realigned and corrected for slice timing before being co-registered to the functional template first and finally to the anatomical template. Images were then smoothed using a spatial Gaussian kernel with a full-width at half-maximum of $2 \mathrm{~mm}^{3}$.

\section{General Linear Model}

Voxel-wise statistics were computed by fitting a general linear model (GLM) to the blood oxygen level-dependent signal. The model contained 3 main regressors, representing the 3 experimental conditions: symmetry (rotation for experiment 1 and reflection for experiment 2), control, and blank periods. These regressors were convolved with the hemodynamic response function estimated from each of the 2 monkeys (see details in Héjja-Brichard et al. 2020). To eliminate noise in our recordings, we performed a principal component analysis on voxels located outside the brain (see Vanduffel and Farivar 2014). Time-courses in those voxels mostly reflect artifacts caused by movement of 
A.

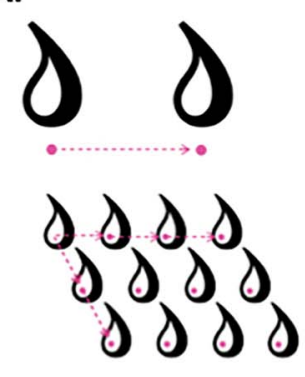

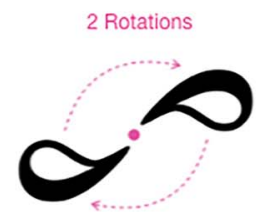

Q $\infty$ \&

3 Rotations

4 Rotations

6 Rotations

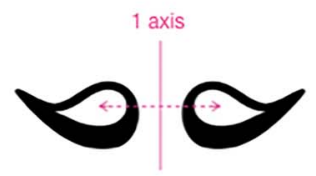

B.

P2

P3

P4
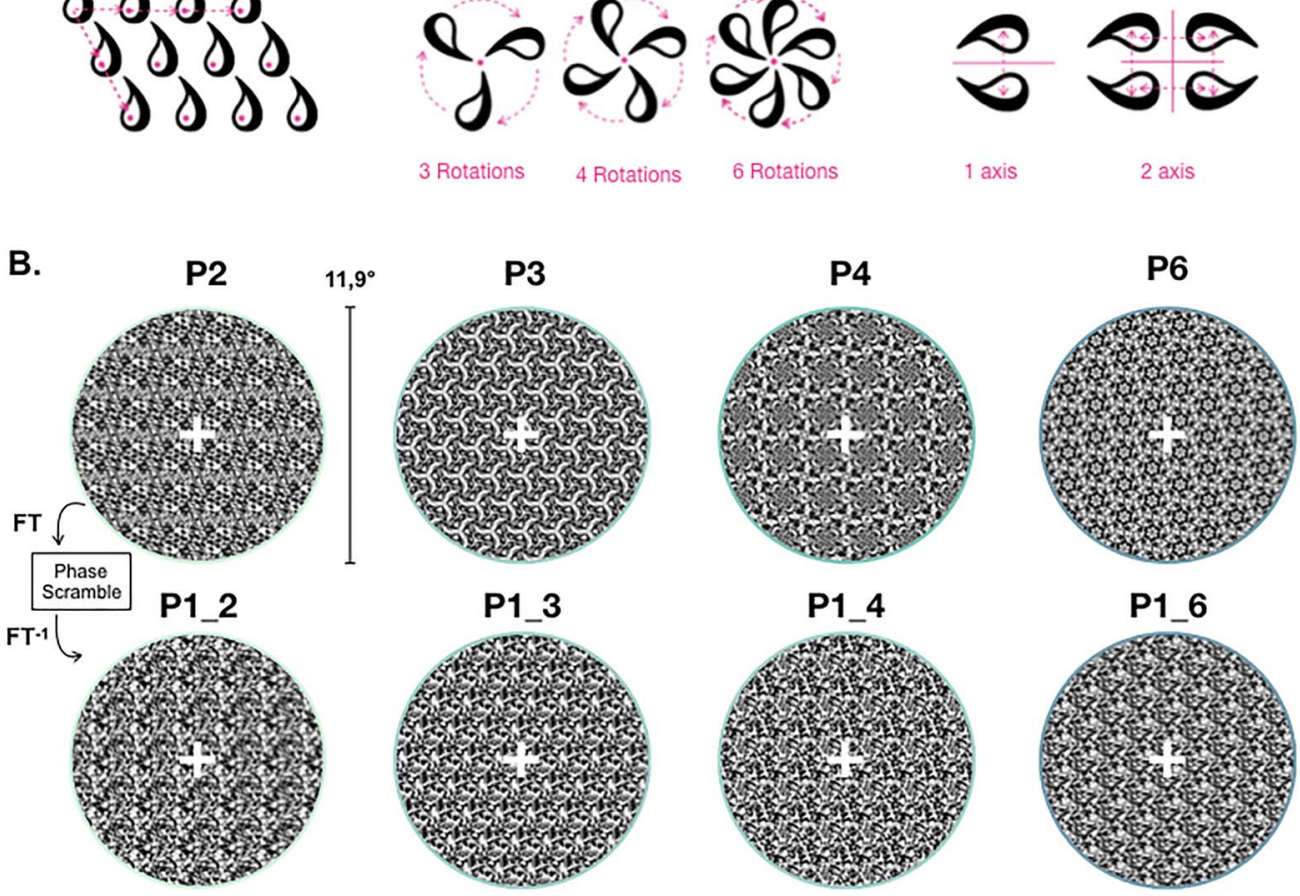

P1_3

P1_4
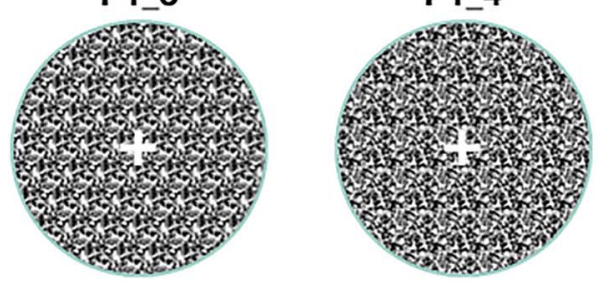

P1_6

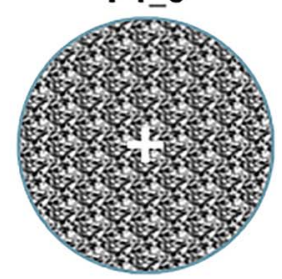

c.

PM_h

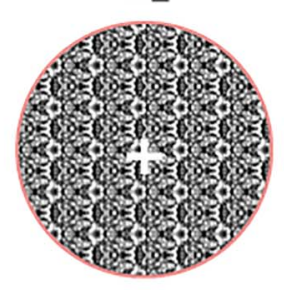

PM_v

PMM

P1_Mh
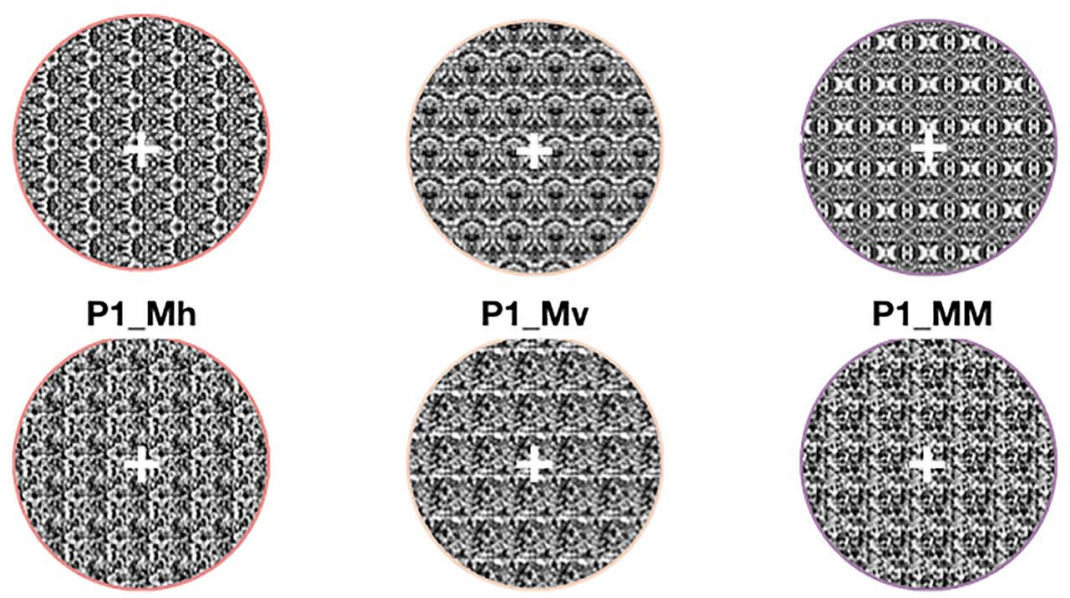

P1_Mv

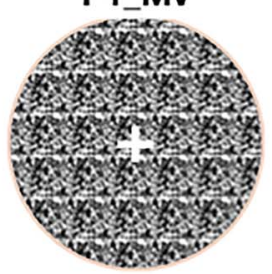

P1_MM

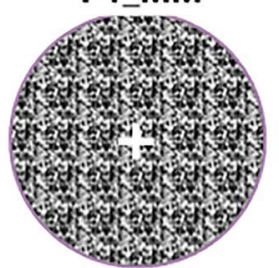

Figure 1. Visual stimuli. (A) Illustrations of the different forms of symmetry embedded in the stimuli: translations (left), rotations of different order (middle), and reflections around 1 (vertical or horizontal) or 2 (both) axes (right). (B) Exemplar images with rotation symmetry (experiment 1) of orders 2 (P2), 3 (P3), 4 (P4), and 6 (P6). For each of these examples, a control condition (P1) was defined by scrambling the phases of the stimulus in the Fourier domain. Each stimulus and its control have the same power spectrum and therefore share the same low-level properties. They both contain translation symmetry but rotation symmetry is no longer present in the control. The white cross represents the fixation target. (C) Exemplar images with reflection symmetry (experiment 2) and their respective controls. Reflection symmetries were based on horizontal axes (PM_h), vertical axes (PM_v), or a combination of both (PMM). Here as well, each stimulus and its control share the same low-level properties and contain translation symmetry, but reflection symmetry is absent in the control. 
the animals and should be independent of our experimental design. For each run, we determined the number of principal components that were necessary to explain $80 \%$ of the variance in these voxels and used the corresponding principal vectors as regressors of noninterest in our model. The beta weights obtained from the GLM were subsequently used to perform univariate analyses (t-scores) at the whole-brain level. These analyses were performed on the preprocessed EPI data and both the beta weights and the associated t-scores were then projected onto the high-resolution volumes of our 2 animals. They were also projected on the individual cortical surfaces (see Figs $2 \mathrm{~A}, 3 \mathrm{~A}, 4 \mathrm{~A}$, and $5 \mathrm{~A}$ ) and on the cortical surface of the F99 template (see Supplementary Fig. 2) using the Caret software (Van Essen et al. 2001). All the preprocessing and GLM analyses were executed using the Matlab and SPM12 softwares.

\section{Analysis in retinotopically defined region-of-interest (ROIs)}

For our 2 animals, we used a population receptive field analysis to define visual areas V1, V2, V3, V3A, and V4 on the individual cortical surfaces from the data collected during an independent wide-field retinotopic experiment (see Héjja-Brichard et al. 2020; Rima et al. 2020). The same data were used to define the MT and PIP clusters and their satellite subregions (V4t, MT, MSTv, and FST for the MT cluster and CIP1, CIP2, PIP1, and PIP2 for the PIP cluster). Because the field of view used for the symmetry experiment $\left(11.9^{\circ} \times 11.9^{\circ}\right.$, see above) was much smaller than during our retinotopic mapping procedure, we restricted these areas and clusters by keeping only the cortical nodes with significant visual responses during the symmetry experiment (i.e., nodes with $t$-scores $>3$ for the contrast between vision and baseline). This operation prevents from including nodes whose receptive fields are outside the stimulated area in the analyses. For each of our 2 experiments, this was done using the data from the other experiment, which means that the restriction of the ROIs was done independently from the data to be analyzed.

Within each of these restricted retinotopic ROIs and clusters, we computed the average beta values for each experimental run and each monkey (data from both hemispheres were combined). These average values were used to perform ROI-level statistical analyses: For each experiment, we estimated the t-scores between the betas obtained in all the symmetry conditions (P2, P3, P4, and P6 for experiment 1, PM_h, PM_v, and PMM for experiment 2) and those obtained in the associated control conditions. Note that in the corresponding figures (Figs $2 B$ and $4 B$ ), we show these data averaged across the 2 monkeys. We also provide $t$-scores in the subparts of the ROIs corresponding to the left and right hemispheres of M01 and M02 to demonstrate their very good correspondence and hence illustrate the robustness and reproducibility of our data. The significance of this correspondence was statistically controlled between hemispheres and animals using correlation analyses performed across all the visual areas. We also performed this test between the t-scores measured in the 2 experiments.

\section{Comparisons between Responses to Different Symmetry Conditions}

In humans, some visual areas have rotation symmetry responses that are proportional to the symmetry order (Kohler et al. 2016). To test whether such properties also exist in macaque, we computed for all our ROIs the percentages of signal change
(PSCs) between each rotation symmetry condition (P2, P3, P4, and P6) and their respective controls (see Fig. 3B). We subsequently performed linear regressions between these PSCs and symmetry order for each monkey using the $\operatorname{lm} 1$ package in $\mathrm{R}$ ( $\mathrm{R}$ Core Team 2014). We considered that a ROI had rotation symmetry responses significantly proportional to the symmetry order only when we found a significant $(P$-value $<0.05)$ linear relationship for both monkeys. We chose this conservative criterion to avoid false positives. PSCs were also used to compare the responses to the different reflection symmetry conditions (experiment 2). These comparisons were performed using 2tailed nonparametric permutation analyses. Here as well, we considered that responses in an ROI were significantly stronger for one condition than for another when significant statistical differences ( $P$-value $<0.05$ ) were found in both the 2 monkeys.

The same permutation analyses were performed to compare the activations obtained in the 2 experiments (rotation and reflection symmetry). However, because the 2 datasets were collected separately, we took into account their possible difference in variability by performing the permutations on normalized data obtained by dividing each PSC by its standard deviation across runs for each condition.

\section{Analyses in ROIs Defined from a Probabilistic Atlas}

In order to characterize activations beyond retinotopic area V4, we used the probabilistic maps of area V4A, dorsal occipitotemporal area (OTd), and ventral and dorsal posterior inferotemporal areas (PITv and PITd) provided by Janssens and Vanduffel (see Janssens et al. 2014) in the CARET software. For each of these areas, we selected the nodes of the F99 template with a probability score above 0.8 and projected the associated binary maps on the inflated reconstructions of the left and right cortical hemispheres of our 2 monkeys (M01 and M02).

\section{Results}

The aim of this study was to characterize the cortical areas that process different forms of symmetry in macaque. We recorded fMRI activations to rotation (experiment 1 ) and reflection (experiment 2) symmetries in 2 awake behaving animals (M01 and M02) involved in a passive fixation task. Our fMRI contrasts were based on control stimuli defined from a phase scrambling in the Fourier domain (Fig. 1A, see also Kohler et al. 2016). This operation modifies the symmetry properties without affecting the amplitude spectrum: Each stimulus and its control share the same low-level properties and contain translation symmetry, but there is no rotation or reflection symmetry in the control (see the Materials and Methods section). We used a blocked design during which periods of visual stimulation (with either symmetric stimuli or their phase-scrambled controls) were interleaved with periods of fixation on a gray screen.

\section{Experiment 1: Responses to Rotation Symmetry}

The experimental design was similar to that used in a previous human fMRI study (Kohler et al. 2016), allowing a direct comparison of the cortical networks processing rotation symmetry in the 2 primate species. We first examined the differences in BOLD responses evoked by the rotation symmetry stimuli (all orders pooled together) and by their respective phase-scrambled controls (see Fig. 1A). Figure 2A presents the corresponding statistical parametric maps (t-scores) projected on dorsal and lateral 
A.

M01
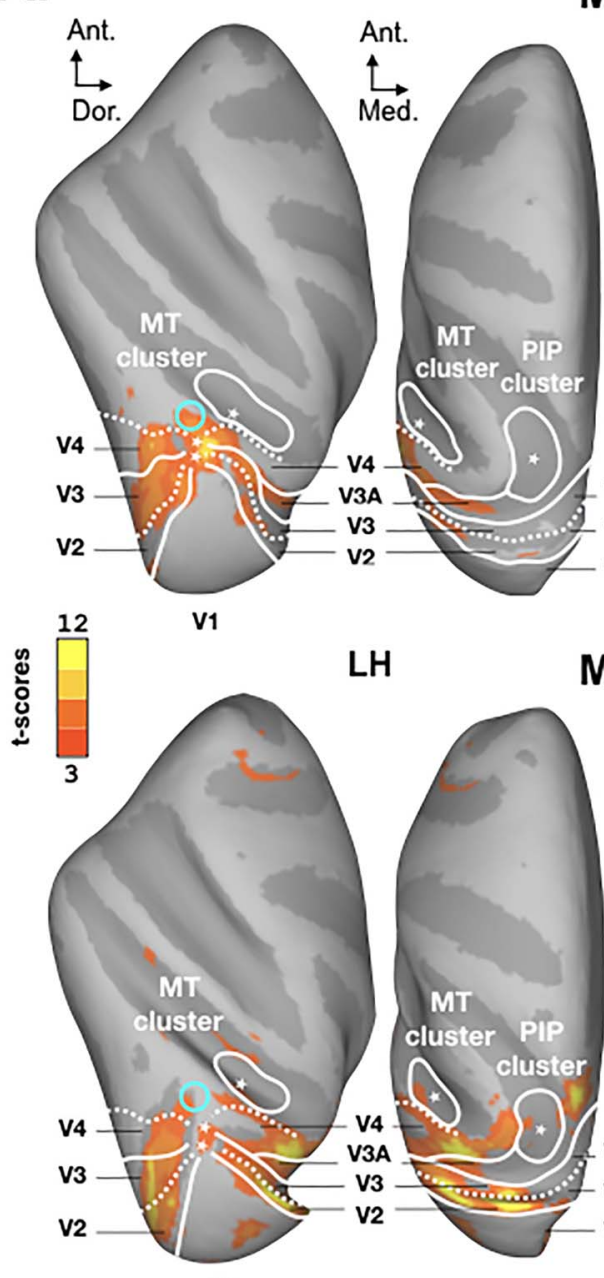

$\mathrm{LH}$

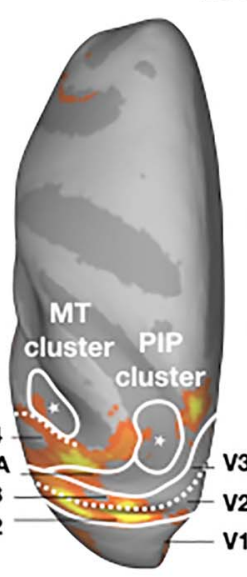

v1

\section{M01}
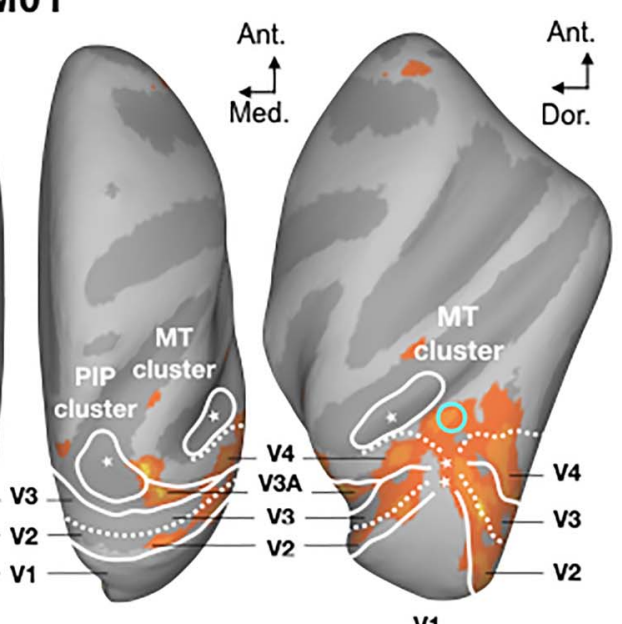

RH

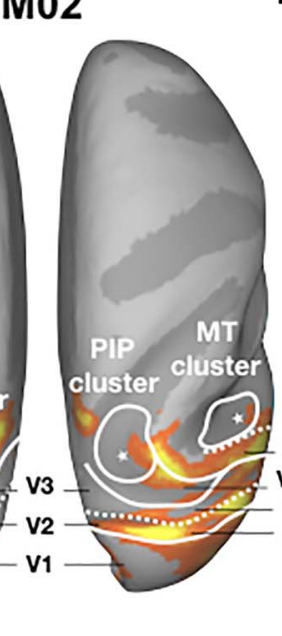

B.

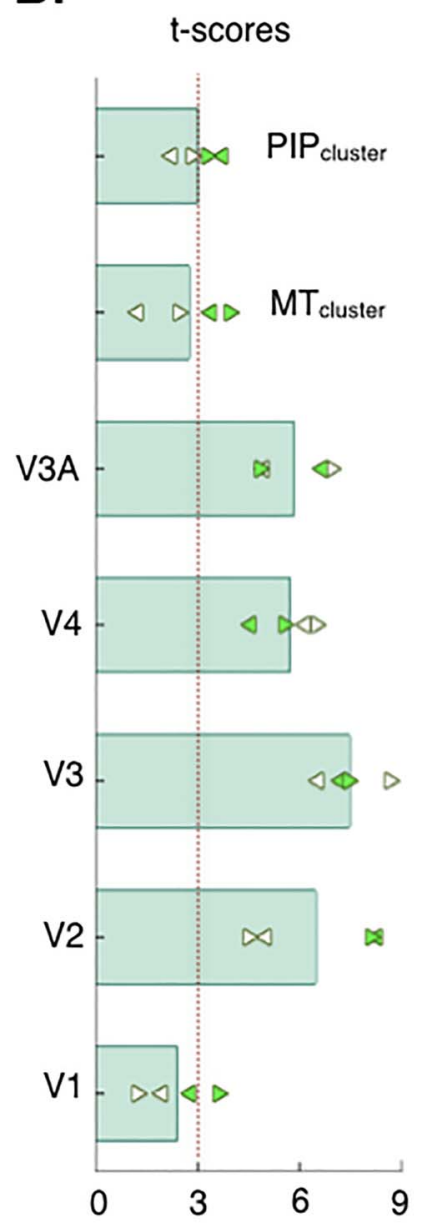

Figure 2. Comparison between BOLD responses to the rotation symmetry (all orders) and to the control conditions. (A) Whole-brain univariate statistical maps. Activations that were stronger for symmetry were projected on the individual cortical surface (dorsal and lateral views) of M01 (upper panel) and M02 (lower panel). Data were thresholded at $\mathrm{P}$-value $<10^{-3}$ (uncorrected). Limits between visual areas (V1, V2, V3, V4, and V3A) obtained from an independent retinotopic mapping protocol are marked by solid (representation of a vertical meridian of the visual field) and dotted (representation of a horizontal meridian) white lines. The MT and PIP clusters (also defined from the retinotopic mapping protocol) are provided by solid white contours. Foveal confluences are marked by stars. Cyan circles show activations beyond retinotopic areas. Ant., anterior; Dor., dorsal; Med., medial; MT, middle temporal; PIP, posterior intra-parietal, LH, left hemisphere; RH, right hemisphere. (B) ROI-based statistics. T-scores for the symmetry versus control conditions within areas V1, V2, V3, V4, and V3A and within the MT and PIP clusters. These values were averaged across the 2 monkeys and are given by the green bars. Left and right arrows provide t-scores in the left and right hemispheres for M01 (white arrows) and M02 (green arrows). The red dotted line corresponds to the threshold ( $t$-score $=3$ ) used in $(A)$.

views of inflated reconstructions of the left and right cortical hemispheres of our 2 monkeys (M01 and M02). Projections on ventral and medial views, for which we did not observe significant activations, are provided in Supplementary Figure 1A. Hot colors (orange to yellow) indicate significantly stronger BOLD activation for symmetry (t-score $>3, P$-value $<0.001$ uncorrected). Very similar activation patterns were observed in the visual cortices of our 2 animals (activation overlaps on the F99 macaque template are provided in Supplementary Fig. 2A). A set of retinotopic areas was independently delineated for each monkey (see Héjja-Brichard et al. 2020; Rima et al. 2020) and overlaid on the activation map. This retinotopic parcellation reveals sensitivity to rotation symmetry in visual areas V2, V3, V4, and V3A. This result was confirmed by ROI-based analyses in all the retinotopically defined areas, including those of the middle temporal (MT) and posterior intra-parietal (PIP) clusters (see Fig. 2B, values in the satellite areas of the MT and PIP clusters are provided in Supplementary Fig. 3A). T-score values in V2, V3, V4, and V3A were consistently greater than 3 for both animals and in both hemispheres. Correlation analyses (see the Materials and Methods section) confirmed that these t-scores were very similar between hemispheres $(R=0.82, P$ value $<0.0001)$ and animals $(R=0.69, P$-value $=0.008)$. Beyond retinotopic areas, stronger responses to symmetry were also observed within the inferotemporal gyrus, anterior to area V4 (see the cyan circles), as described in detail below.

\section{Effects of Rotation Symmetry Order}

In humans, some visual areas exhibit BOLD responses proportional to the rotation symmetry order (Kohler et al. 2016). In order to test whether such an effect also exists in macaque, we first 
A.

M01

M02

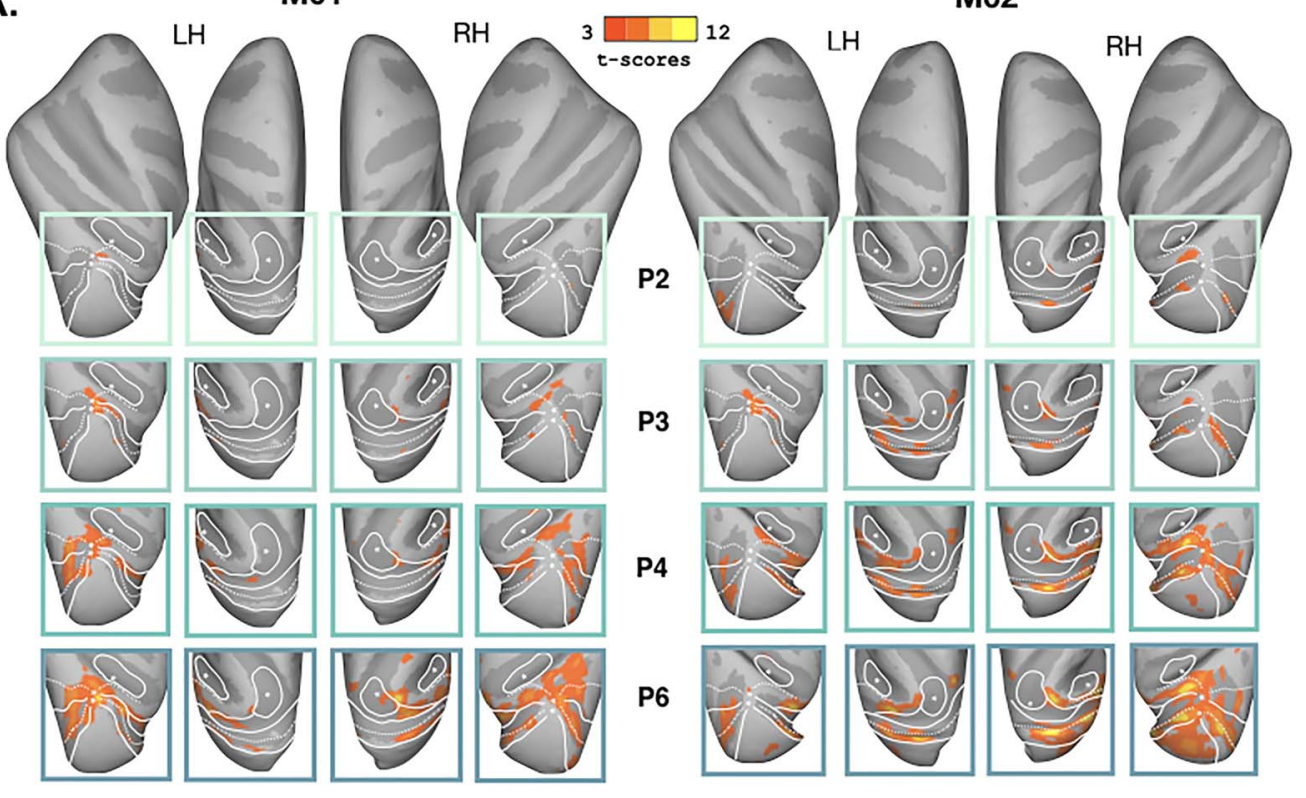

B.
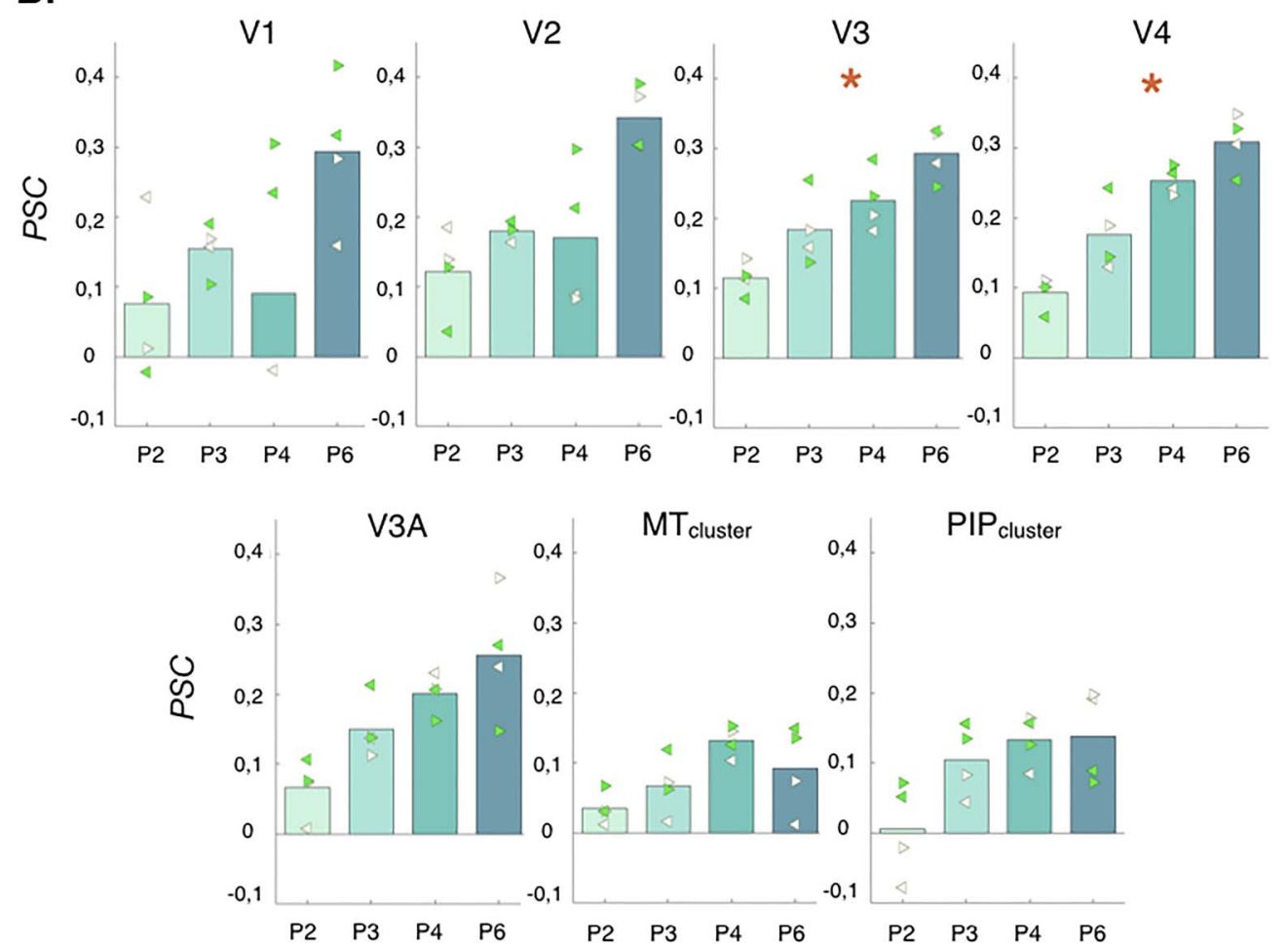

Figure 3. Effects of rotation symmetry order on BOLD responses. (A) Whole brain univariate statistical maps. Activations that were stronger for each symmetry order (P2, P3, P4, and P6) with respect to their control conditions are shown in the different boxes for M01 (leftward columns) and M02 (rightward columns). Data were thresholded at P-value $<10^{-3}$ (uncorrected). See Figure 2A for more details. (B) PSCs between the responses to the rotation symmetry conditions (P2, P3, P4, and P6) and those to their respective controls. Data are shown in retinotopic areas (V1, V2, V3, V3A, and V4) and in the MT and PIP clusters. Left and right arrows provide values in the left and right hemispheres for M01 (white arrows) and M02 (green arrows). Areas marked with a star ("*") are those for which we found a significant linear relationship between PSCs and symmetry order in both the 2 animals. 
examined the whole brain statistical maps corresponding to the difference in BOLD signal between the 4 wallpaper groups (P2, P3, P4, and P6) and their respective controls (see Fig. 3A). There is a clear tendency for activations to increase with symmetry order in the left and right hemispheres of both monkeys. Next, we computed the corresponding PSC for all retinotopic ROIs (including the MT and PIP clusters). These values are shown in Figure 3B (values in the areas constituting the MT and PIP clusters are provided in Supplementary Fig. 4A, individual data for M01 and M02 are shown in Supplementary Fig. 5).

We also performed linear regressions between these PSCs and the symmetry orders, independently for each animal and for each ROI (averaged across hemispheres). We found significant linear relationships in both monkeys for areas V3 ( $t$-score $=3.181$, $P$-value $=0.0022$ in $\mathrm{M} 01$ and $t$-score $=2.53, P$-value $=0.0130$ in M02) and V4 (t-score $=4.073, P$-value $=0.0001$ in M01 and t-score $=3.030, P$-value $=0.0031$ in $\mathrm{M02}$ ). The corresponding equations and the variances explained by these linear models are provided in Supplementary Table 1. Importantly, we verified that these parametric effects were driven by an increase in the responses to the different symmetry conditions and not caused by variations in the responses to their control conditions. Finally, we also found significant linear relationships in V2 for M02 and in V3A for M01.

\section{Experiment 2: Responses to Reflection Symmetry}

Next, we characterized fMRI responses to images containing different axes and amounts of reflection symmetry. Stimuli were wallpaper groups that included reflection symmetry with horizontally oriented axes (PM_h), vertically oriented axes (PM_v), or both (PMM) (see Fig. 1B). We first examined BOLD signal differences between the responses evoked by the symmetry stimuli (all conditions pooled together) and their P1 controls. Figure 4A presents the corresponding statistical parametric maps (t-scores) projected on dorsal and lateral views for M01 and M02 (projections on ventral and medial views are provided in Supplementary Fig. 1B). Response patterns are very similar between the 2 animals (see the overlapping map in Supplementary Fig. 2B) and also match very closely those observed for rotation symmetry (overlaps between the 2 types of symmetry are provided in Supplementary Fig. 2C).

As was the case for rotation, the most responsive areas are V2, V3, V4 and V3A with t-scores greater than 3 in the 2 hemispheres of the 2 monkeys (see the ROI-based statistics in Fig. 4B, t-scores in the satellite areas of the MT and PIP clusters are provided in Supplementary Fig. 3B). Correlation analyses confirmed that $t$-scores were very similar between hemispheres $(R=0.647, P$-value $<0.0001)$ and animals $(R=0.872$, $P$-value $=0.0001$ ). They also demonstrated a very good correspondence with the $t$-scores obtained in the first experiment $(R=0.9204, P$-value $<0.0001)$. Beyond retinotopic areas, stronger responses to reflection symmetry were also observed within the inferotemporal gyrus, anterior to area V4 (see the cyan circles).

\section{Effects of the Axes of Symmetry}

In the first experiment, we found that some visual areas exhibit BOLD responses proportional to the rotation symmetry order. For reflection symmetry, responses could therefore be more pronounced for conditions with more symmetry axes (i.e., in the PMM condition). In order to test this hypothesis, we examined the statistical maps corresponding to the difference in BOLD signal between each reflection symmetry condition (PM_h, PM_v, and PMM) and their respective controls (see Fig. $5 \mathrm{~A}$, only dorsal and lateral views are shown here, ventral and medial views are provided in Supplementary Fig. 1B). Responses are generally more pronounced for the PMM condition. The corresponding PSCs in the retinotopic ROIs and in the MT and PIP clusters are provided in Figure 5B (values within the areas of the MT and PIP clusters are provided in Supplementary Fig. 4B, individual data for M01 and M02 are shown in Supplementary Fig. 6)

Nonparametric permutation tests demonstrated that stimuli containing both horizontal and vertical symmetries (PMM) elicited significantly stronger responses than those with only horizontal symmetries (PM_h) for both monkeys in areas V3 $(P$-value $=0.0021$ in $\mathrm{M} 01$ and $P$-value $=0.0038$ in M02), V4 ( $P$ value $=0.0036$ in $\mathrm{M} 01$ and $P$-value $=0.0023$ in M02), and V3A ( $P$ value $=0.0036$ in M01 and $P$-value $<0.0001$ in M02). We also found that PMM stimuli evoked significantly stronger responses than stimuli containing vertical symmetries (PM_v) in areas V3A for M02 and in V3 and V4 for M01.

We also compared the activations obtained in our 2 experiments (rotation and reflection symmetry). This was done on normalized PSCs to take into account the possible difference of variability between the 2 datasets (see the materials and methods section). Supplementary Figure 7A shows these data sorted in ascending order for each visual area. Responses to stimuli containing less symmetry (conditions $\mathrm{P} 2, \mathrm{PM} \_\mathrm{h}$, and $\mathrm{PM} \_\mathrm{v}$ ) are generally weaker than those to stimuli containing more symmetry (conditions PMM and P6) in symmetry selective areas (i.e., in V2, V3, V4, and V3A). Statistical analyses demonstrated that responses to the PMM conditions were significantly higher than those to the P2 condition for both animals in areas V3 $(P$-value $=0.0159$ in M01 and $P$-value $=0.0246$ in M02), V4 ( $P$-value $=0.0167$ in $\mathrm{M} 01$ and $P$-value $=0.0196$ in M02), and V3A ( $P$-value $=0.0153$ in M01 and $P$-value $=0.0182$ in M02).

Finally, to control whether all the symmetry responses and statistical effects reported above were different between central (i.e., near the fixation cross) and peripheral vision, we subdivided each of our visual ROIs by only including cortical nodes whose preferred eccentricity (estimated from our retinotopic data) were below $2.5^{\circ}$ or between $2.5^{\circ}$ and $5.95^{\circ}$ (i.e., the radius of our stimuli). We reran all the analyses and found that our results remained unchanged in both cases. We conclude that symmetry responses are independent of eccentricity in our study.

\section{Symmetry Responses beyond V4 in Atlas-Based ROIs}

For both rotation and reflection symmetries, significant responses were observed beyond V4, within the inferotemporal gyrus (see the cyan circles in Figs $2 \mathrm{~A}$ and $4 \mathrm{~A}$ ). To further characterize these responses, we performed ROI-based analyses in the V4A area, in the OTd, and in the ventral and dorsal posterior inferotemporal areas (PITV and PITd) defined from the probabilistic atlas of Janssens and Vanduffel (see the Materials and Methods section and Janssens et al. 2014). The definition of these ROIs on the inflated reconstructions of the left and right cortical hemispheres of our 2 monkeys are shown in Figure $6 \mathrm{~A}$ (lateral views). T-scores for the 2 types of symmetry are given in Figure $6 \mathrm{~B}$ (t-scores in area V4 defined from the same atlas are also provided for comparison with values in retinotopically 


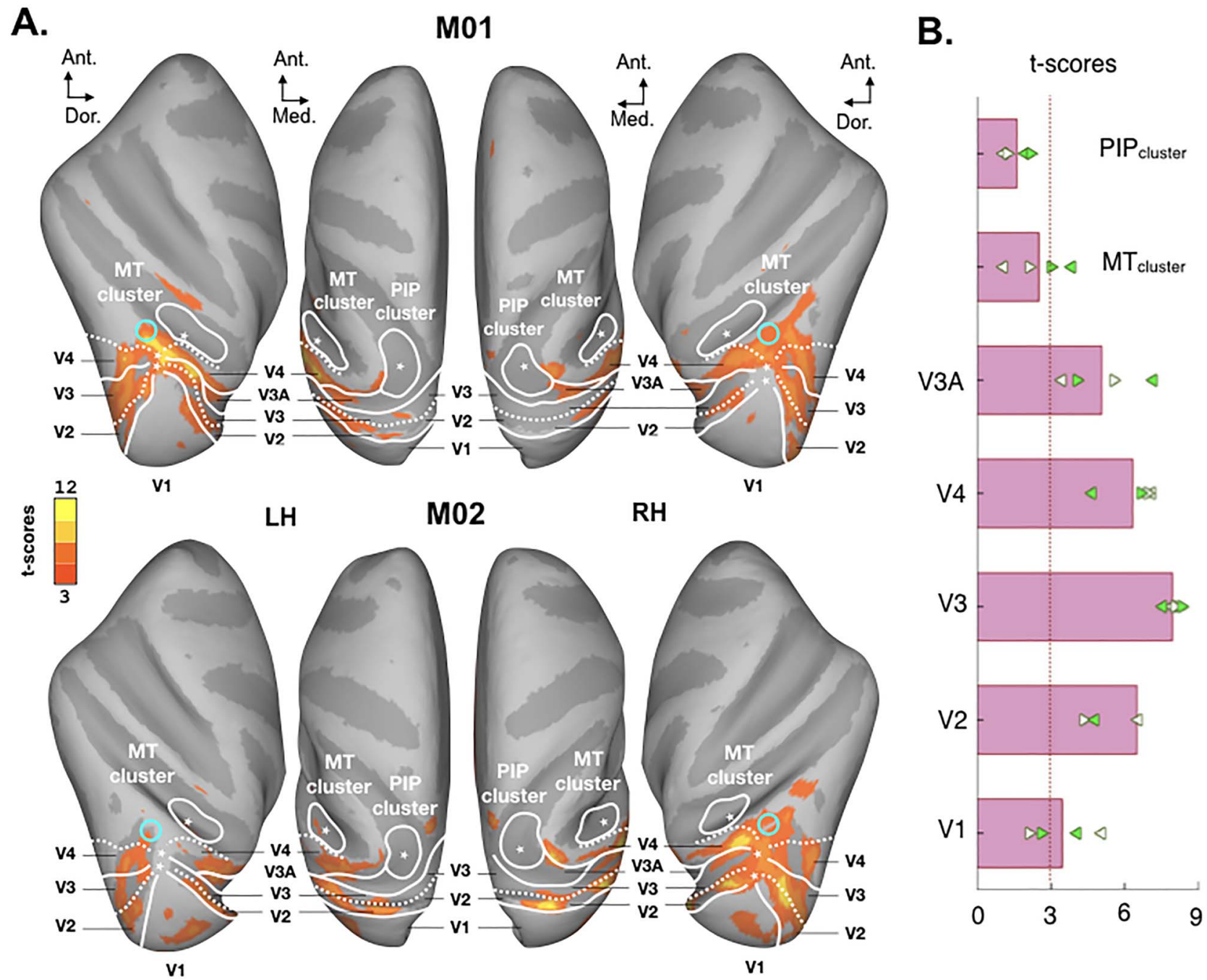

Figure 4. Comparison between responses to reflection symmetry (all conditions) and to the control conditions. (A) Whole brain univariate statistical maps. See Figure $2 \mathrm{~A}$ for more details. (B) ROI-based statistics. See Figure 2B for more details.

defined V4). As for the retinotopically defined ROIs (see Figs $2 B$ and $4 B$ ), there is a very good correspondence between the statistical values obtained for rotation and reflection symmetry. Beyond V4, the most responsive area is $\mathrm{V} 4 \mathrm{~A}$ with $\mathrm{t}$-scores greater than 3 for both experiments. By comparison, symmetry responses in areas OTd, PITv, and PITd are only moderate (t-scores around 3). However, as for areas V3 and V4 (see Fig. 3B), we found a significant linear relationship between PSC and rotation symmetry order for area PITd for both animals (t-score $=2.17, P$-value $=0,033$ in $\mathrm{M} 01$ and $t$-score $=2.82$, $P$-value $=0,005$ in M02, see Fig. 6C and Supplementary Table 1 for the associated equation and variance explained). Significant linear relationships were also found in area OTd for M01. We did not find significant difference between responses to the different reflection symmetry conditions in the same area for both monkeys (Fig.6D), even though permutation tests showed that responses to PMM were stronger than those to PM_h in area V4A and PITV for M01 and PITd for M02. We showed in Supplementary Figure 7B the normalized PSCs (see the Materials and Methods) obtained for the 2 experiments, sorted in ascending order for areas V4A, OTd, PITd, and PITV.
Statistical analyses demonstrated that, as in areas V3, V4, and V3A (see above), activations in PITd for the PMM condition were significantly higher than those for the P2 conditions in both animals ( $P$-value $=0.0115$ in M01 and $P$-value $=0.0043$ in M02).

\section{Discussion}

The goal of this sensory study was to characterize the areas in macaque cortex that process symmetry and to determine whether these areas have potential counterparts among the set of areas observed in humans (Sasaki et al. 2005; Kohler et al. 2016; Keefe et al. 2018; Van Meel et al. 2019). We recorded fMRI activations to rotation (experiment 1) and reflection (experiment 2) symmetries in 2 awake behaving animals involved in a passive fixation task. Our experimental protocol was directly derived from a recent human study (Kohler et al. 2016) to permit a direct comparison between the 2 primate species. Among the areas defined based on an independent retinotopic mapping experiment, we found that V2, V3, V4, and V3A had significant symmetry selective responses (see Figs 2 and 4). We also found 
A. M01

A.

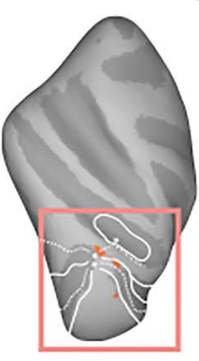

$\mathrm{LH}$
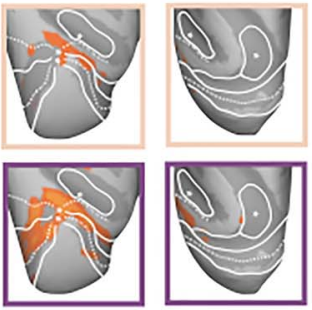
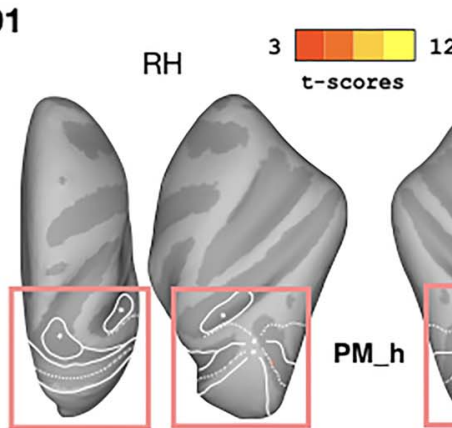

12

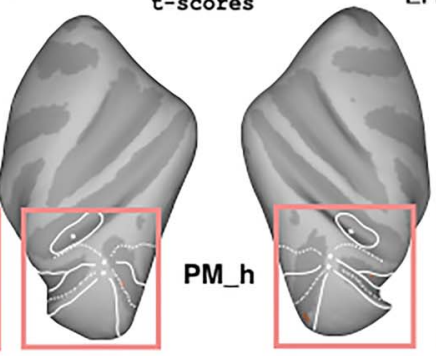

$\mathrm{LH}$
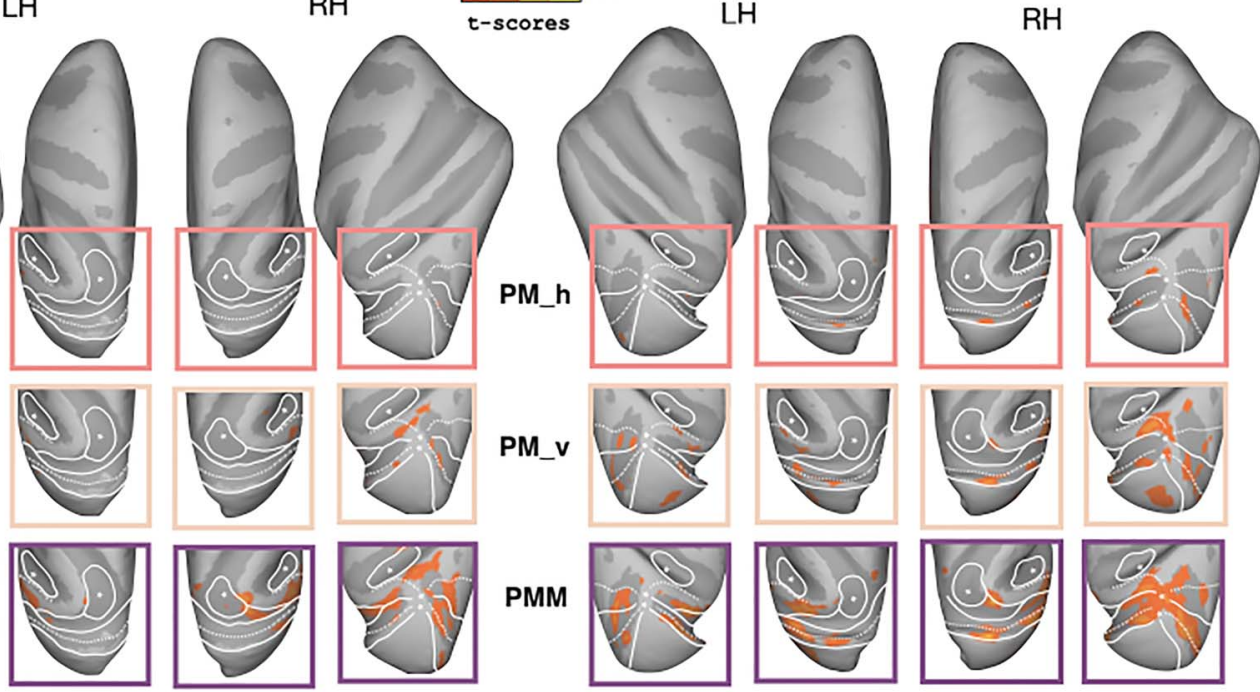

B.
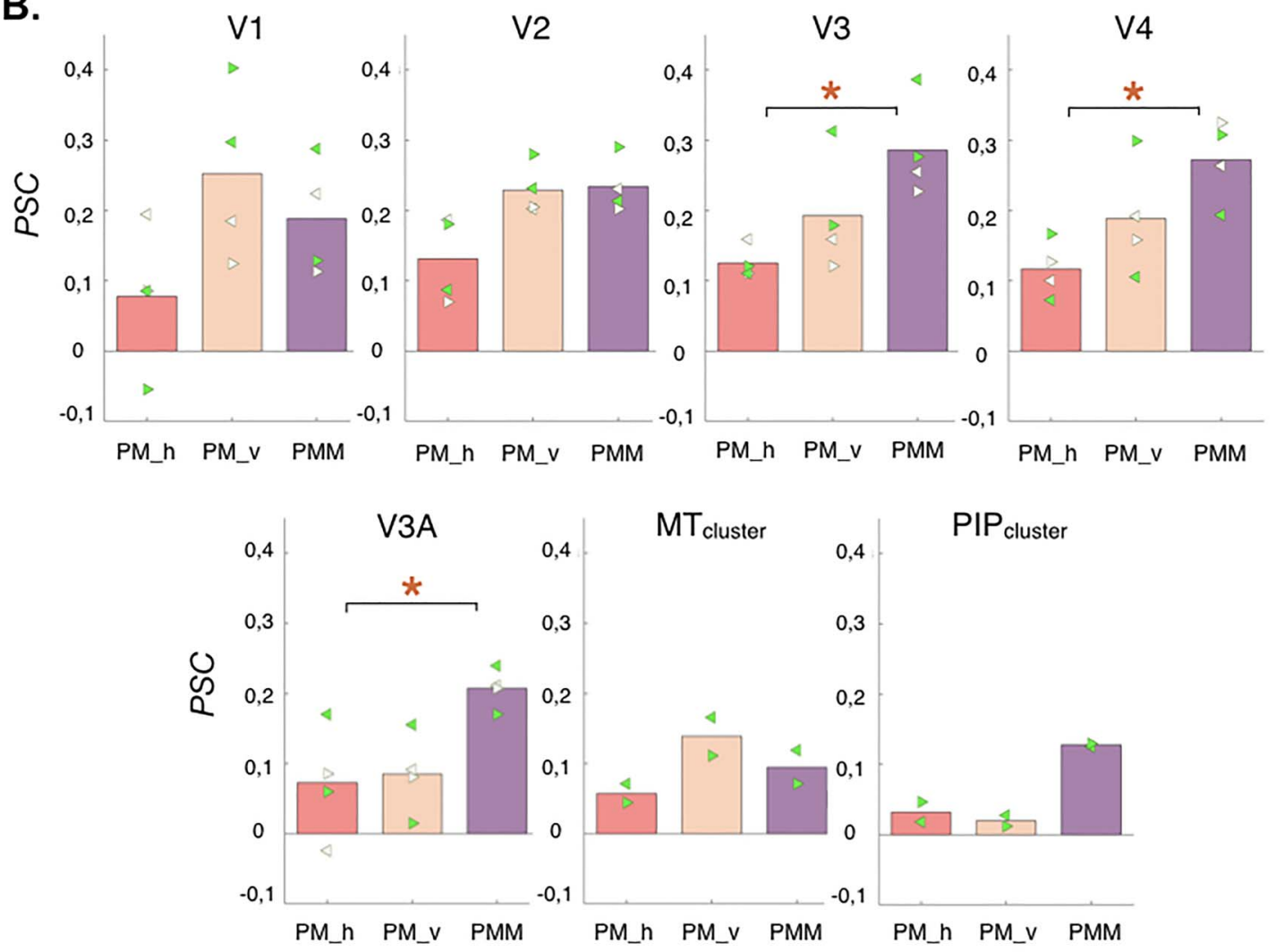

Figure 5. Effects of the different reflection symmetry conditions on BOLD responses. (A) Whole brain univariate statistical maps. Responses that were stronger for each symmetry order (PM_h, PM_v, and PMM) with respect to their control conditions are shown in the different boxes for M01 (leftward columns) and M02 (rightward columns). See Figures $2 \mathrm{~A}$ and $3 \mathrm{~A}$ for more details. (B) PSCs obtained for each of the reflection symmetry conditions (PM_h, PM_v, and PMM) versus their respective controls. See Figure $3 B$ for more details. Stars indicate ROIs for which the nonparametric permutation tests found that PMM elicited significantly stronger responses than PM_h in both of the 2 animals.

symmetry-related activations beyond V4 in a location corresponding to V4A as identified using a probabilistic atlas (see Fig. 6). Interestingly, response levels to rotation and reflection symmetry in all these areas were highly correlated, which suggests similar cortical processing for these 2 types of symmetry in macaques. We observed parametric responses to rotation symmetry in areas V3, V4, and PITd and higher responses for reflection symmetry around 2 axes rather than one in V3, V4, and V3A. We also found higher activations for reflection symmetry around 2 axes than for rotation symmetry of order 2 in 
A.

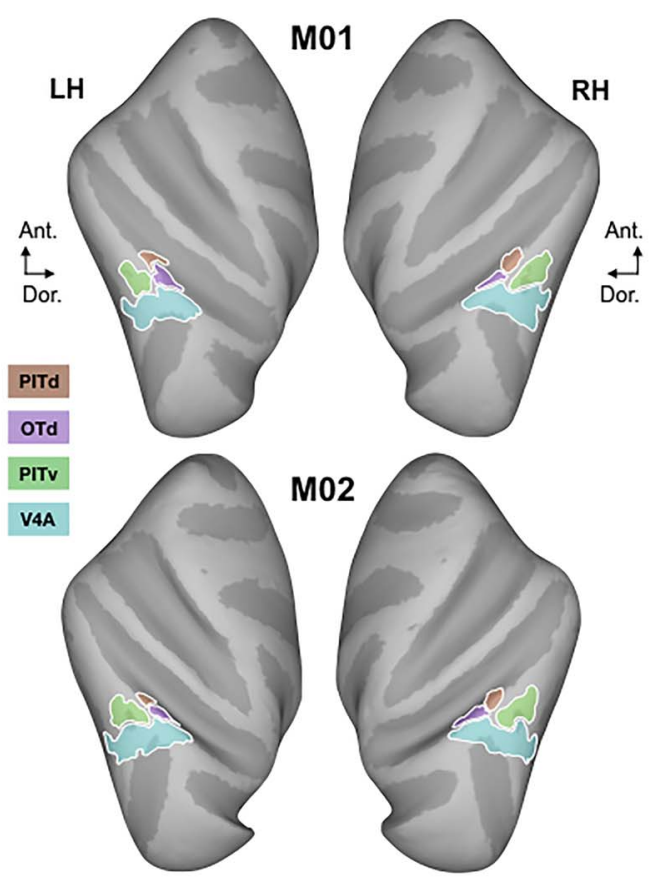

B.

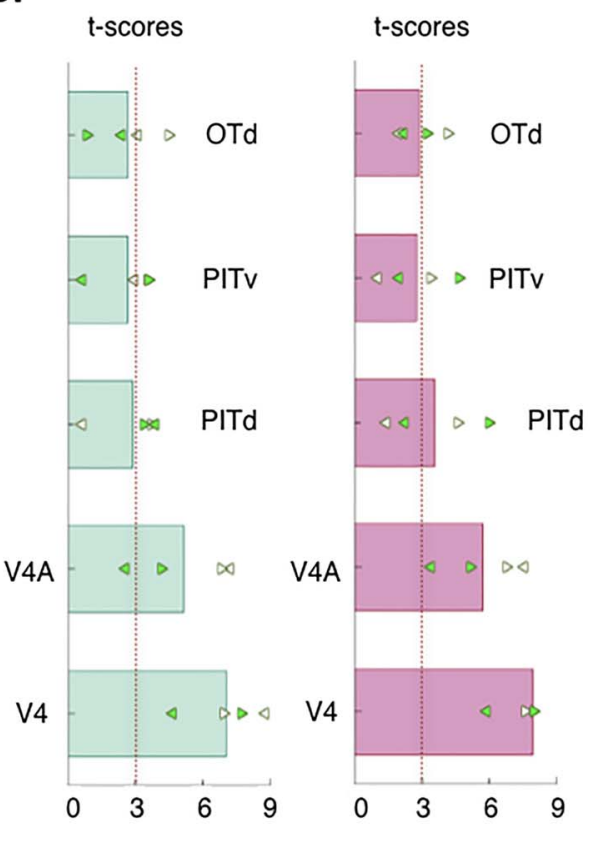

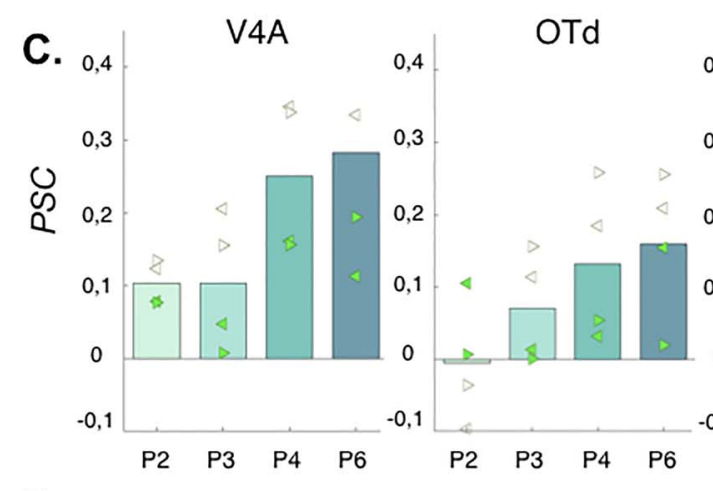

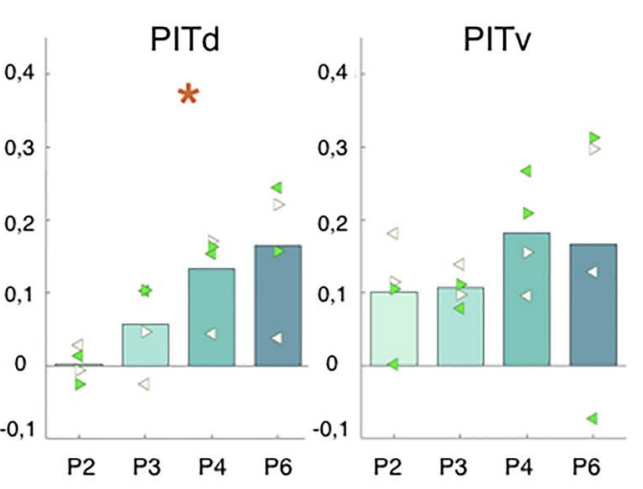

D.
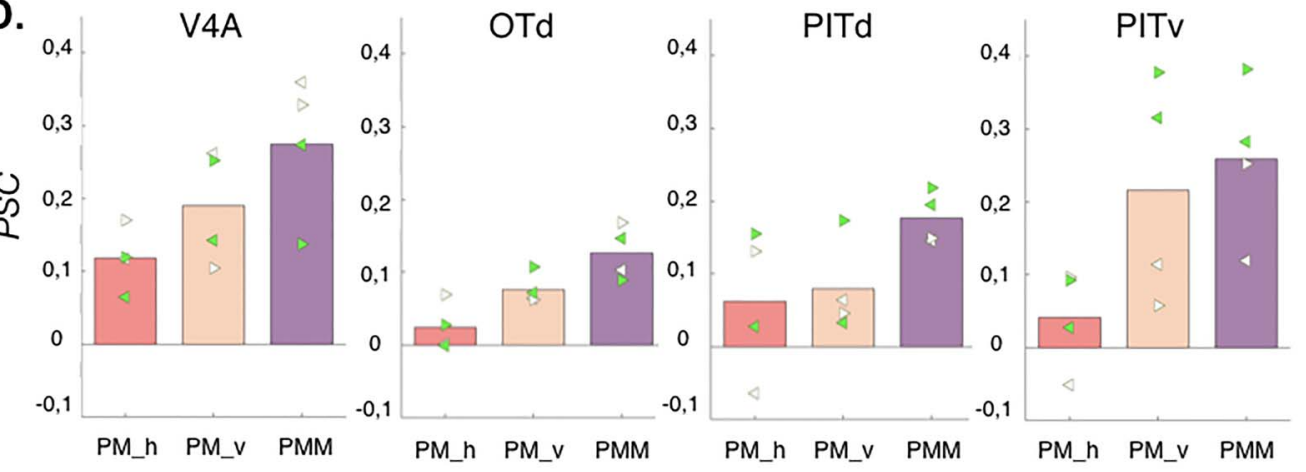

Figure 6. (A) Areas V4A, OTd, PITv, and PITd defined from the probabilistic atlas of Janssens and Vanduffel and shown on lateral views of inflated reconstructions of the left and right cortical hemispheres of our 2 monkeys (M01 and M02). (B) T-scores for the symmetry versus control conditions within these areas. Values for rotation (experiment 1) and reflection (experiment 2) symmetries are respectively shown on the left and on the right. Values were averaged across the 2 monkeys. Left and right arrows provide t-scores in the left and right hemispheres for M01 (white arrows) and M02 (green arrows). (C) PSCs between the responses to the rotation symmetry conditions (P2, P3, P4, and P6) and those to their respective controls. Left and right arrows provide values in the left and right hemisphere for M01 (white arrows) and M02 (green arrows). Areas marked with a star (“*”) are those for which we found a significant linear relationship between PSCs and symmetry order in both the 2 animals. (D) PSCs obtained for each of the reflection symmetry conditions (PM_h, PM_v, and PMM) versus their respective controls. See (C) for more details. 
areas V3, V4, V3A, and PITd. We further discuss the implications of these results below.

Only one previous fMRI study explored symmetry responses in macaques using reflection symmetry patterns defined by random dots (Sasaki et al. 2005). The authors found significant activations in a cortical network which was restricted to areas V4d and V3A and much less extended than the one observed in humans using the same stimuli. They concluded that symmetry processing is generally weaker in monkeys than in humans. If our results are compatible with this previous study because we also found significant symmetry responses in macaque areas V4 and V3A, they reveal a much larger cortical network. As we will discuss below, this network overlaps well with what has been observed in humans under the same experimental conditions (see Kohler et al. 2016). Our study therefore points toward a different interpretation and suggests that symmetry processing might be similar between the 2 primate species, at least when symmetry is embedded in regular textures like the ones used here. Importantly, the more extended activations we observed are unlikely to reflect an improvement in signal sensitivity because we used standard BOLD measurements at 3 Teslas whereas Sasaki et al. (2005) used contrast agents or high-field fMRI (7 Teslas). They are more likely a result of the fact that we used textures in which symmetric patterns were repeated within small unit lattices whereas Sasaki et al. (2005) used random dots with overall reflection symmetry around the vertical and/or horizontal meridians.

In our data, BOLD responses to rotation and reflection symmetry patterns were consistent in area V2 (t-scores $>3$ ), whereas they were marginal in V1. However, we did not observe significant parametric modulation of $\mathrm{V} 2$ responses with rotation symmetry order (experiment 1, Fig. 3B) nor stronger responses for reflection symmetry around 2 axes rather than one (experiment 2, Fig. 5B). These results suggest that macaque $\mathrm{V} 2$ is responsive to rotation and reflection symmetry but that these responses are less sophisticated than in other areas (see below). The increased sensitivity to image structure in V2 is consistent with a previous study, which found that single-cell responses in V2, but not in V1, were sensitive to synthetic stimuli replicating the higherorder statistical dependencies found in natural texture images (Freeman et al. 2013). In humans, V2 has generally not been found to be responsive to symmetry, although Van Meel et al. (2019) reported a higher interhemispheric connectivity in this area during symmetry perception.

Responses to rotation and reflection symmetry were strong in V3. This is in line with previous findings in humans for rotation (Kohler et al. 2016) and for reflection (Sasaki et al. 2005; Keefe et al. 2018; Van Meel et al. 2019) symmetry. In our data, V3 was the earliest area in the macaque visual processing stream with parametric responses to rotation symmetry (Fig. 3B). Using the same protocol as was used in our first experiment, Kohler et al. (2016) also found strong parametric responses to rotation symmetry in human V3, but not V2. In our second experiment, we found that V3 responses were significantly higher for reflection symmetry around 2 axes (i.e., in the PMM condition) rather than one (Fig. 5B). Finally, we also found that V3 responses were significantly higher for reflection symmetry around 2 axes (i.e., in the PMM condition of experiment 2) than for rotation symmetry of order 2 (i.e., in the P2 condition of experiment 1, see Supplementary Fig. 7A). This is interesting given that the P2 symmetry group is a subgroup of the PMM group (see Kohler and Clarke 2021). Altogether, these results suggest that macaque $\mathrm{V} 3$ is sensitive to the amount of (rotation and/or reflection) symmetry present in the stimuli. V3 receives inputs from V1 and V2 and projects notably to V4 (Felleman et al. 1997; Gegenfurtner et al. 1997). Using the same stimuli as in the present study, an EEG source localization approach in humans (Kohler et al. 2016) has suggested that given the latencies of symmetry responses in V3, activations in this area are unlikely to reflect feedback from higher cortical areas downstream the visual pathway. In both humans and macaques, V3 could therefore constitute an important step for the feedforward integration of symmetry and more generally of forms and textures (see Felleman et al. 1997 for a characterization of V3 responses to higher-order forms). Area V3 is often omitted in the current models of visual processing along the ventral stream (see Riesenhuber and Poggio 2000 or DiCarlo and Cox 2007). Our results suggest that it plays an important role in sophisticated form processing typically ascribed to the ventral stream and calls for its incorporation in future models.

Symmetry responses were also strong in area V4 and they share the properties already observed in V3. This is not surprising given that macaque V4 is known to process forms (Desimone and Schein 1987) and textures (Okazawa et al. 2015). Many commonalities exist between human and monkey V4 (Roe et al. 2012), and accordingly, our results are in agreement with the fMRI activations observed in humans for rotation (Kohler et al. 2016) and reflection (Sasaki et al. 2005; Keefe et al. 2018; Van Meel et al. 2019) symmetry in this area. In both species, V3 and V4 could realize an intermediate processing of symmetric patterns before more sophisticated treatments in downstream areas of the ventral pathway. Indeed, human studies consistently reported that areas VO1 (and VO2) and object selective regions like the lateral occipital complex (LOC) play an important role in symmetry perception (Bona et al. 2014; Kohler et al. 2018). In macaque, our analyses in regions V4A and PITd defined from probabilistic maps (Fig. 6) showed that area V4A (a potential homologous of human VO1, see Arcaro and Livingstone 2017) had significant responses to both rotation and reflection symmetry (t-scores $>3$ ). PITd, which is strongly activated by shapes and forms (Kolster et al. 2014), as human LOC, had parametric responses to rotation symmetry. It also had higher responses for reflection symmetry around 2 axes (i.e., in the PMM condition of experiment 2) than for rotation symmetry of order 2 (i.e., in the P2 condition of experiment 1).

In the dorsal pathway, we found significant symmetry activations in macaque V3A, in agreement with all the previous studies which explored reflection symmetry in its human counterpart (Sasaki et al. 2005; Keefe et al. 2018; Van Meel et al. 2019). Reflection responses in this area were significantly higher for reflection symmetry around 2 axes (i.e., in the PMM condition) rather than one. V3A responses to reflection symmetry around 2 axes were also significantly higher than those to rotation symmetry of order 2 . We did not observe parametric responses to rotation symmetry order, which is also in line with the human results of Kohler et al. (2016). This result suggests an interesting distinction between the ventral and dorsal pathways with only the former having parametric responses to rotation symmetry.

Like its human counterpart (Kohler et al. 2016) and previous monkey experiments (Sasaki et al. 2005), this study focused on sensory brain responses and did not explore perception. Behavioral approaches might be useful in the future to determine how the cortical responses reported here can be related to symmetry perception. Symmetry is usually believed to contribute to viewpoint invariant representations of objects (Li et al. 2013) and to 
the encoding of regularity and structure in visual scenes (Liu et al. 2010; Kohler et al. 2016; Kohler and Clarke 2021). The fact that the human and macaque visual systems carry parametric responses to rotation and reflection symmetry suggests that representations of symmetry are much richer and more detailed than previously believed. In humans, recent results suggest that this high level of encoding actually extends to nearly every combination of symmetries in the 2D plane and can be read out using both brain imaging and psychophysical measures (Kohler and Clarke 2021). Our finding that macaques share these detailed representations and that corresponding visual areas are involved in monkeys and humans is critically important because it eliminates the possibility that the responses are partly driven by human aesthetic preferences. Instead, it suggests a general sensory mechanism that could be shared across the 2 primate species. The findings open the door to further research into the role of symmetry in pattern and texture perception during everyday vision.

In conclusion, our results suggest that the cortical networks that process rotation and reflection symmetry in humans and macaques are rather similar. They call for a deeper exploration of their functional homologies in future studies and open the door to a characterization of the underlying neural mechanisms at the cellular level, notably in area V3, where a parametric dependency on symmetry order was observed.

\section{Supplementary Material}

Supplementary material can be found at Cerebral Cortex online.

\section{Funding}

This research was supported by a grant from the 'Agence Nationale de la Recherche' (ANR-16-CE37-0002-01, ANR JCJC 3D3M) awarded to Benoit R. Cottereau. It was also supported in part by the Vision Science to Applications (VISTA) program funded by the Canada First Research Excellence Fund (CFREF, 2016-2023) and by a Discovery Grant from the Natural Sciences and Engineering Research Council of Canada awarded to Peter J. Kohler.

\section{Notes}

We thank the Inserm/UPS UMR1214 Technical Platform for the MRI acquisitions. We also thank the Animalliance staff for their help with monkey welfare. P.J.K. acknowledges funding support from the Vision Science to Applications (VISTA) program funded by the Canada First Research Excellence Fund (CFREF, 20162023) and from the National Sciences and Engineering Research Council of Canada. Conflict of Interest: The authors declare no conflicts of interest.

\section{References}

Arcaro MJ, Livingstone MS. 2017. Retinotopic organization of scene areas in macaque inferior temporal cortex. J Neurosci. 37(31):7373-7389. 10.1523/JNEUROSCI.0569-17.2017.

Bertamini M, Silvanto J, Norcia AM, Makin ADJ, Wagemans J. 2018. The neural basis of visual symmetry and its role in midand high-level visual processing: neural basis of visual symmetry. Ann N Y Acad Sci. 1426(1):111-126. 10.1111/nyas.13667.

Bona S, Herbert A, Toneatto C, Silvanto J, Cattaneo Z. 2014. The causal role of the lateral occipital complex in visual mirror symmetry detection and grouping: an fMRI-guided TMS study. Cortex. 51:46-55. 10.1016/j.cortex.2013.11.004.

Cottereau BR, Smith AT, Rima S, Fize D, Héjja-Brichard Y, Renaud L, Lejards C, Vayssière N, Trotter Y, Durand J-B. 2017. Processing of egomotion-consistent optic flow in the rhesus macaque cortex. Cereb Cortex. 27(1):330-343. 10.1093/cercor/bhw412.

Delius JD, Nowak B. 1982. Visual symmetry recognition by pigeons. Psychol Res. 44(3):199-212. 10.1007/BF00308420.

Desimone R, Schein SJ. 1987. Visual properties of neurons in area V4 of the macaque: sensitivity to stimulus form. J Neurophysiol. 57(3):835-868. 10.1152/jn.1987.57.3.835.

DiCarlo JJ, Cox DD. 2007. Untangling invariant object recognition. Trends Cogn Sci. 11(8):333-341. 10.1016/j.tics.2007.06.010.

Fedorov ES. 1891. The symmetry of regular systems of figures (in Russian). Proceedings of the Imperial St. Petersburg Mineralogical Society. 28:1-146.

Felleman DJ, Burkhalter A, Van Essen DC. 1997. Cortical connections of areas V3 and VP of macaque monkey extrastriate visual cortex. J Comp Neurol. 379(1):21-47. 10.1002/(sici)10969861(19970303)379:1<21::aid-cne3>3.0.co;2-k.

Felleman DJ, Van Essen DC. 1991. Distributed hierarchical processing in the primate cerebral cortex. Cereb Cortex. 1(1):1-47. 10.1093/cercor/1.1.1.

Freeman J, Ziemba CM, Heeger DJ, Simoncelli EP, Movshon JA. 2013. A functional and perceptual signature of the second visual area in primates. Nat Neurosci. 16(7):974-981. 10.1038/nn.3402.

Gegenfurtner KR, Kiper DC, Levitt JB. 1997. Functional properties of neurons in macaque area V3. J Neurophysiol. 77(4):1906-1923. 10.1152/jn.1997.77.4.1906.

Giurfa M, Eichmann B, Menzel R. 1996. Symmetry perception in an insect. Nature. 382(6590):458-461. 10.1038/382458a0.

Héjja-Brichard Y, Rima S, Rapha E, Durand J-B, Cottereau BR. 2020. Stereomotion processing in the nonhuman primate brain. Cereb Cortex. 30(8):4528-4543. 10.1093/cercor/bhaa055.

Jacobsen T, Höfel L. 2003. Descriptive and evaluative judgment processes: behavioral and electrophysiological indices of processing symmetry and aesthetics. Cogn Affect Behav Neurosci. 3(4):289-299. 10.3758/CABN.3.4.289.

Janssens T, Zhu Q, Popivanov ID, Vanduffel W. 2014. Probabilistic and single-subject retinotopic maps reveal the topographic organization of face patches in the macaque cortex. J Neurosci. 34(31):10156-10167. 10.1523/JNEUROSCI.291413.2013.

Keefe BD, Gouws AD, Sheldon AA, Vernon RJW, Lawrence SJD, McKeefry DJ, Wade AR, Morland AB. 2018. Emergence of symmetry selectivity in the visual areas of the human brain: fMRI responses to symmetry presented in both frontoparallel and slanted planes. Hum Brain Mapp. 39(10):3813-3826. 10.1002/hbm.24211.

Kohler PJ, Clarke A, Yakovleva A, Liu Y, Norcia AM. 2016. Representation of maximally regular textures in human visual cortex. J Neurosci. 36(3):714-729. 10.1523/JNEUROSCI.2962-15.2016.

Kohler PJ, Clarke ADF. 2021. The human visual system preserves the hierarchy of two-dimensional pattern regularity. Proc $R$ Soc B. 288(1955):20211142. 10.1098/rspb.2021.1142.

Kohler PJ, Cottereau BR, Norcia AM. 2018. Dynamics of perceptual decisions about symmetry in visual cortex. Neuroimage. 167:316-330. 10.1016/j.neuroimage.2017.11.051.

Kolster H, Janssens T, Orban GA, Vanduffel W. 2014. The retinotopic organization of macaque occipitotemporal cortex 
anterior to V4 and caudoventral to the middle temporal (MT) cluster. J Neurosci. 34(31):10168-10191. 10.1523/JNEUROSCI.3288-13.2014.

Li Y, Sawada T, Shi Y, Steinman RM, Pizlo Z. 2013. Symmetry is the sine qua non of shape. In: Dickinson SJ, Pizlo Z, editors. Shape perception in human and computer vision. London: Springer London, pp. 21-40 (last accessed 1 September 2021).

Lightman AP. 2013. The accidental universe: the world you thought you knew. 1. American ed. New York: Pantheon Books.

Liu Y, Hel-Or HS, Kaplan C, Van Gool L. 2010. Human visual perception of two-dimensional symmetry. PsyArXiv (last accessed 1 March 2021). https://osf.io/x7uvf.

Makin AD, Rampone G, Pecchinenda A, Bertamini M. 2013. Electrophysiological responses to visuospatial regularity. Psychophysiology. 50(10):1045-1055. 10.1111/psyp.12082.

Makin ADJ, Rampone G, Wright A, Martinovic J, Bertamini M. 2014. Visual symmetry in objects and gaps. J Vis. 14(3):12-12. 10.1167/14.3.12.

McLaren DG, Kosmatka KJ, Kastman EK, Bendlin BB, Johnson SC. 2010. Rhesus macaque brain morphometry: a methodological comparison of voxel-wise approaches. Methods. 50(3):157-165. 10.1016/j.ymeth.2009.10.003.

McLaren DG, Kosmatka KJ, Oakes TR, Kroenke CD, Kohama SG, Matochik JA, Ingram DK, Johnson SC. 2009. A populationaverage MRI-based atlas collection of the rhesus macaque. Neuroimage. 45(1):52-59. 10.1016/j.neuroimage.2008.10.058.

Merry J, Morris MR. 2001. Preference for symmetry in swordtail fish. Anim Behav. 61(2):477-479. 10.1006/anbe.2000.1589.

Møller AP. 1992. Female swallow preference for symmetrical male sexual ornaments. Nature. 357(6375):238-240. 10.1038/357238a0.

Okazawa G, Tajima S, Komatsu H. 2015. Image statistics underlying natural texture selectivity of neurons in macaque V4. PNAS. 112(4):E351-E360. 10.1073/pnas.1415146112.

Orban GA, Van Essen D, Vanduffel W. 2004. Comparative mapping of higher visual areas in monkeys and humans. Trends Cogn Sci. 8(7):315-324. 10.1016/j.tics.2004.05.009.

R Core Team. 2014. R: A language and environment for statistical computing. Vienna, Austria: R Foundation for Statistical Computing. http://www.R-project.org/.
Riesenhuber M, Poggio T. 2000. Models of object recognition. Nat Neurosci. 3(S11):1199-1204. 10.1038/81479.

Rima S, Cottereau BR, Héjja-Brichard Y, Trotter Y, Durand J-B. 2020. Wide-field retinotopy reveals a new visuotopic cluster in macaque posterior parietal cortex. Brain Struct Funct. 225(8):2447-2461. 10.1007/s00429-020-02134-2.

Roe AW, Chelazzi L, Connor CE, Conway BR, Fujita I, Gallant JL, Lu H, Vanduffel W. 2012. Toward a unified theory of visual area V4. Neuron. 74(1):12-29. 10.1016/j.neuron.2012.03.011.

Sasaki Y, Vanduffel W, Knutsen T, Tyler C, Tootell R. 2005. Symmetry activates extrastriate visual cortex in human and nonhuman primates. Proc Natl Acad Sci U S A. 102(8):3159-3163. 10.1073/pnas.0500319102.

Van Essen DC, Lewis JW, Drury HA, Hadjikhani N, Tootell RBH, Bakircioglu M, Miller MI. 2001. Mapping visual cortex in monkeys and humans using surface-based atlases. Vision Res. 41(10-11):1359-1378. 10.1016/S0042-6989(01)00045-1.

Van Essen DC. 2002. Surface-based atlases of cerebellar cortex in the human, macaque, and mouse. Ann N Y Acad Sci. 978:468-479. 10.1111/j.1749-6632.2002.tb07588.x.

Van Meel C, Baeck A, Gillebert CR, Wagemans J, Op de Beeck HP. 2019. The representation of symmetry in multi-voxel response patterns and functional connectivity throughout the ventral visual stream. Neuroimage. 191:216-224. 10.1016/j.neuroimage.2019.02.030

Vanduffel W, Farivar R. 2014. Functional MRI of awake behaving macaques using standard equipment. In: Papageorgiou TD, Christopoulos GI, Smirnakis SM, editors. Advanced brain neuroimaging topics in health and disease-methods and applications. InTech. http://www.intechopen.com/books/adva nced-brain-neuroimaging-topics-in-health-and-diseasemethods-and-applications/functional-mri-of-awake-beha ving-macaques-using-standard-equipment (last accessed 2 March 2021).

Wagemans J. 1997. Characteristics and models of human symmetry detection. Trends Cogn Sci. 1(9):346-352. 10.1016/S1364-6613(97)01105-4.

Waitt C, Little AC. 2006. Preferences for symmetry in conspecific facial shape among Macaca mulatta. Int J Primatol. 27(1):133-145. 10.1007/s10764-005-9015-y. 